\title{
Sensor Data Collection Using Heterogeneous Mobile Devices
}

\author{
Prem Prakash Jayaraman*, Arkady Zaslavsky*, Jerker Delsing** \\ \{prem.jayaraman, arkady.zaslavsky\}@infotech.monash.edu.au,jerker.delsing@ltu.se \\ * Monash University, Melbourne, Australia \\ ** Lulea University of Technology, Lulea, Sweden
}

\begin{abstract}
Data collection has always been a major challenge in sensor networks and various techniques have been proposed to enable efficient data collection. One such methodology is the use of mobile elements within the existing infrastructure to enable data collection. The paper proposes the use of existing mobile elements like mobile phones which have enough spare capacity to act as data carriers within a sensor network to carry sensor data. With advent of technology, mobile devices have become so powerful that they can work in a pervasive environment and make decisions based on context information like presence, location etc. Our proposal is an intelligent heterogeneous network in which the sensor nodes act as the data accumulators and the context-aware mobile phones act as data carriers of the sensed data. A framework that enables the mobile node and sensor node communication over Bluetooth is proposed and a $\mathrm{p}$ implementation is presented.
\end{abstract}

\section{INTRODUCTION}

Wireless sensor nodes are gaining more and more popularity with increased amounts of research in this area due to their versatile list of application. The sensor nodes are power constrained small sensing devices capable of measuring and monitoring the environment [1]. Recent advancement in technology has increased the popularity of mobile devices like mobile phones, personal digital assistant, smart phones etc. These devices comprise spare resources for processing, and communication that can be used as a bridge to enable sensor data collection. These powerful mobile devices are context aware, hence forming a intelligent heterogeneous network as depicted in Figure 1 in which data generators namely sensor nodes work at a lower level and intelligent data collectors act as data carriers for the sensor data.

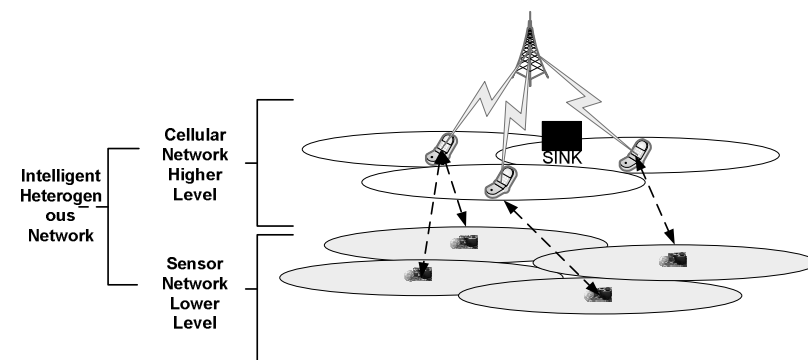

Figure 1: Intelligent Heterogeneous Network

Here by context we represent mobile devices context including current location, future location and direction of travel. This context information can be used by the mobile device to compute a cost efficient data collection, negotiation and delivery strategy. Hence we look into harvesting existing mobile infrastructure as an access network for cost efficient sensor data transmission. Here the cost includes the cost involved in communication, power spent on communication by the sensor node, time involved in data delivery and the infrastructure setup cost.

This paper proposes data collection within sensor nodes using context aware mobile elements in the surrounding as data carriers to form an intelligent heterogeneous network. These mobile elements are existing elements and are not introduced to enable sensor data collection. Lot of research has gone into exploring the use of mobile elements for data collection. But our approach makes use of a more novel technique where context aware pervasive devices are used as data carriers.

\section{DATA COLLECTION - AN OVERVIEW}

A sensor network is a data centric network which comprises distributed sites of information represented by sensor nodes contributing data sensed [2]. They are producer of valuable data that needs to be collected in a cost efficient manner. The cost includes cost of communication, power consumption and time involved in communication. Data collection can be classified into Data Collection using static nodes and Data Collection using mobile elements. Since our focus is on mobile device based data collection, the literature looks into the use of mobile elements for data collection and not into traditional based multi hop and clustering based techniques.

The idea of using mobility within sensor network to collect data from sensor nodes has been discussed by a number of researchers $[3,4,5,6,7,8,9,10]$. The use of mobility to improve performance in Ad-Hoc networks has been proposed in ZebraNet project and Manatee project [3]. These mobile elements can be introduced or existing mobility in the environment can be used to achieve mobility. The use of mobility can be classified broadly as Random mobility, Predictable mobility and Controlled mobility.

Kansal et al. [4, 12] presents a work on using controlled mobility for data collection in sensor networks. Shah et al. [3] and Sushant et al [12] present Data Mules that makes use of random mobility for data collection. Chakrabarthi et al. [11] proposes a novel way of using predictable mobility to collect data from sensor networks that are distributed over an area. 


\section{DESIGN CONSIDERATIONS}

Our proposal of using mobile phones as data collectors is motivated by the fact that mobile phones have enough spare capacity for processing and communicating. Hence they can be used as a means to collect sensor data and transfer it to the sink. To use mobile nodes as a shared network resource, the mobile nodes need to be context aware, enabling them to communicate with sensor nodes, negotiate and deliver data to the sink. The context aware mobile nodes work within an environment, which, we describe as a virtual personal area network (VPAN) [13]. The VPAN as depicted in Figure 2 depicts VPAN, our vision of the future. A VPAN is an imaginary boundary within which the devices are location and context aware and can communicate with any other device irrespective of the type of the device.

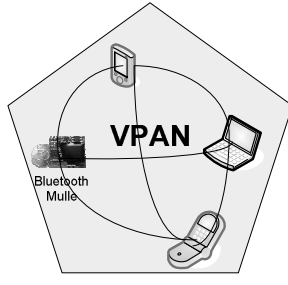

Figure 2: Virtual Personal Area Network (VPAN) [13]

The context in the VPAN is assumed to be a distributed context, part of which, is available on the mobile node and the rest is available within the VPAN environment. The system architecture proposed in the paper focuses mainly on the mobile nodes system framework which enables it to communicate with the sensor node and make decisions on sensor data collection and delivery.

\section{A. System Framework}

Figure 3 illustrates the architecture of the proposed system. The context part proposed in the paper is a preliminary step towards the use of context information to enable the mobile node to obtain current location and environment information. The external context server is a context server external to the system that is accessible to the mobile node as long as it is within the VPAN. The in build tiny context has presence and user calendar information from which it can get information on user's next possible location and his presence information i.e. if the user is where he is supposed to be.

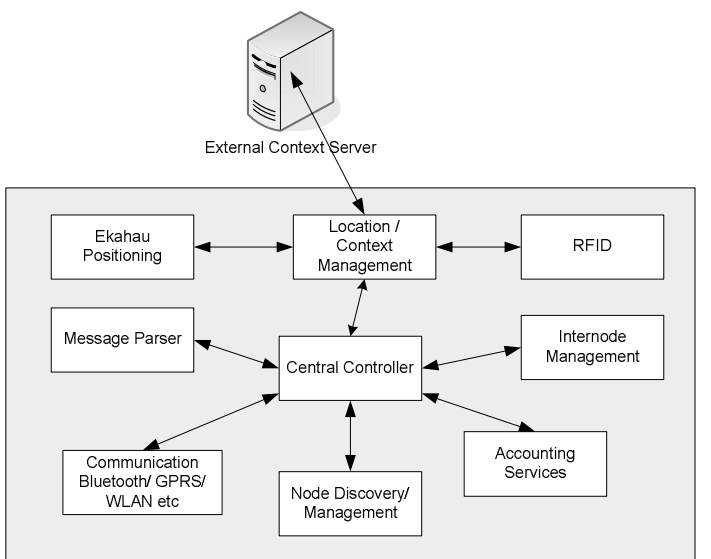

Figure 3: Architecture of the system on the mobile node
The message parser is responsible to parse messages that are sent between the mobile node and the sensor node and between the mobile nodes. There are two types of messages, control messages and data. Figure 4 shows the format of the control and data messages respectively.

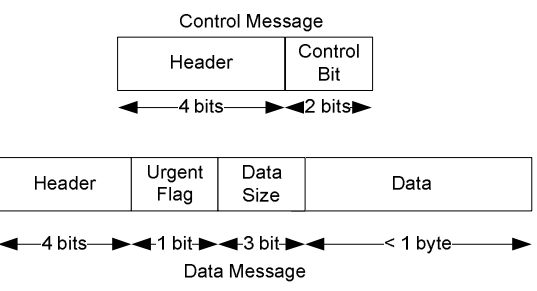

Figure 4: Message Format

The node discovery and management is responsible to discover nodes in the surrounding and store the address of any node that communicates with the mobile node along with its location and the activation schedule. This is useful since, the next time the mobile node is in the same location, it can decide to communicate with the sensor node if the sensor node responds. The pseudo code in Figure 5 illustrated the node discovery and management.

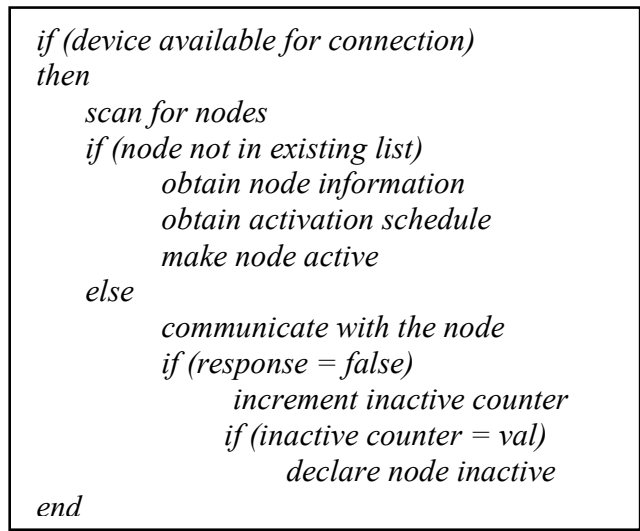

Figure 5: Node Discovery and Management

\section{IMPLEMENTAION}

The proposed architecture is a framework for further development. To prove that the model is feasible, we have implemented a prototype implementation that makes use of the framework to enable mobile nodes to collect data from sensor nodes and deliver it to a sink. Since our implementation is dependent on context, we take into consideration specific scenario where augmentation of context is more feasible.

The sensor node that we chose for our implementation is Mulle [14] a COST sensor network node developed at the EISLAB, Luleå University of Technology, Sweden. The Mulle is a generic wireless sensor node that uses Bluetooth for its communication. The Mulle used for implementation has an onboard temperature sensor. Our proposal of using spare capacity of mobile nodes as a access data network needs to work with sensor nodes that run standardized protocols, so that they can communicate with any mobile device. Since Bluetooth is now a commonly available stack on almost every mobile phone when compared to zigbee [17], we use a sensor node that talks Bluetooth over standard IP based protocols. 


\section{A. Activation schedule}

The Mulle has an activation schedule [15] which it uses to switch between its operational modes namely passive, active and time synchronous. In active mode, the Bluetooth radio is on and listens for incoming connections. In passive mode, it is in sleep mode and hence most power conservative mode. In the timesynchronous mode, the Mulle alternates between the passive and the active mode at specific defined time intervals.

\section{B. Event based connection}

In most cases, periodic transmission of sensor data would not be necessary. Hence we look into an event based approach where we define the event as an activity that that is triggered by the change in the parameter being monitored by the sensor node.

$$
\begin{aligned}
& \Delta \mathrm{T}>\mathrm{Ta}-\alpha \mathrm{T} \\
& \Delta \mathrm{T} \text {-> Max temperature difference } \\
& \mathrm{Ta} \text {-> Actual temperature } \\
& \alpha \mathrm{T} \text {-> Predefined temperature threshold }
\end{aligned}
$$

If the difference in temperature $\Delta \mathbf{T}$ is greater than the pre-defined threshold value Ta - $\alpha \mathrm{T}$, then the Mulle moves from its passive mode to an active mode becoming ready to transmit data. Once a device locates the Mulle, the Mulle transmits the message to the mobile node over a Bluetooth connection. This approach is represented in Figure 6 where the other two connections depict the time synchronous operation of the Mulle where it wakes up every $\mathrm{t}$ seconds to offload data to near by mobile node data carrier.

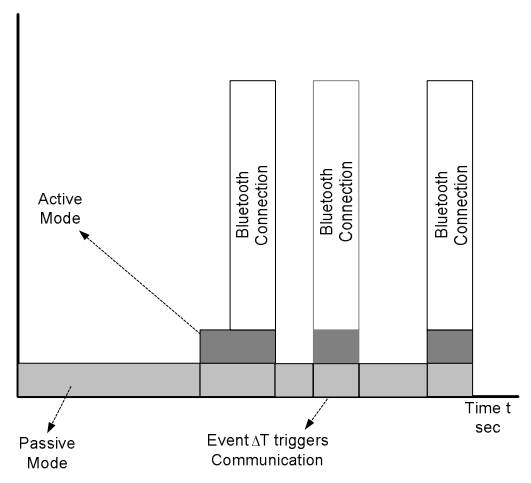

Figure 6: Event based Mulle working

\section{Implementation Scenario and Assumptions}

For implementation purposes, we implemented the Bluetooth Mulle functionalities on a PDA. The PDA based sensor node is very similar to the Bluetooth Mulle since it uses the standard Bluetooth stack with IP based protocols for communication. Since the implementation is a proof of concept, we looked into the challenges of implementing the framework in the mobile node and enhancing the Mulle to facilitate activation schedule and event based connection.

The mobile node that collects data is a PDA based smart phone that can talks to the Mulle over Bluetooth and can use GPRS and WLAN for communication with the sink. The scenario for our implementation is a building environment shown in Figure 7 . Since most buildings infrastructures have WLAN connectivity, we assume that the cost of communication over WLAN is free and hence WLAN is used over GPRS. The sink node is a Bluetooth enabled laptop that can also talk to the mobile nodes over WLAN. Hence urgent messages are sent over WLAN while regular alive messages are sent over Bluetooth. For the current implementation, we consider the delivery of messages over Bluetooth and over the WLAN as free to use communication. Hence the current implementation does not look into the complexities of using an accounting component on the sink to negotiate credits with the mobile node. Both the PDA's run Windows Mobile 2003 edition and the implementation is done using Microsoft .Net Compact Framework 2.0. The PDA is equipped with a class 2 Bluetooth module that has a radius of 10 meters. The sink is a IBM T42 laptop that runs the BlueSoleil stack [16]. It runs the sink software written using Microsoft .NET. The Mulle's activation schedule is implemented in such a way that the node wakes up once in every 4 hours to send alive messages and the value of $\alpha \mathbf{T}$ is set to 23 and $\Delta \mathrm{T}$ is set to 5 .

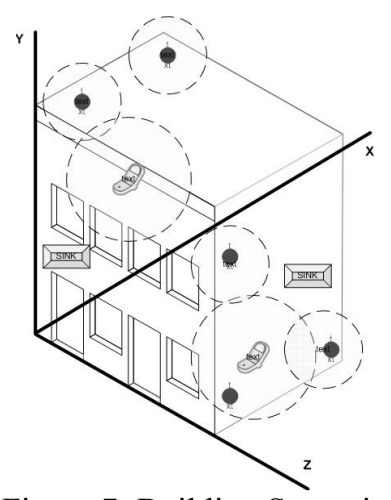

Figure 7: Building Scenario

The Mulle's activation schedule is preloaded. Hence it wakes up at specific time durations to transfer the data to the mobile node. The mobile node which is the PDA smart phone, when in the area, collects context information about the location and the duration of its stay at the location. This is useful since, the mobile node might not want to talk to the sensor node and abruptly disconnect the connection. Once it determines that it can spend sometime to do data collection, it scans the surrounding area for Mulle. The sensor nodes are named as EISMULLE1 to $n$. Hence once the node is discovered, the mobile node communicates with the Mulle using Bluetooth serial port profile. The Mulle negotiates with the mobile node using a 2 bit control message as shown in Figure 8.

$$
\begin{aligned}
& 00 \text {-> Ready } \\
& 01 \text {-> Stop } \\
& 10 \text {-> Start } \\
& 11 \text {-> Wait }
\end{aligned}
$$

Figure 8: Control Bits

The sink can accept multiple connections from mobile nodes. The mobile node offloads the data to the sink. The sink uses the timestamp to sort old sensor data to new ones. For our preliminary test setup, we used two PDA based sensor nodes and one mobile node that moves around collecting data. 


\section{PRELIMINARY RESULTS}

We have computed an energy model that gives the energy spent by the node while transmitting and receiving $\mathrm{T}_{\text {bits }}$ of data over distance $\mathrm{D}$.

Total Transmitting Energy $=\mathbf{f}_{E T X}\left(T_{b i t s}, D\right)+E_{c o n} * R_{c o n}+E_{c p u}$ Total Receiving Energy $=\mathbf{f}_{E R X}\left(T_{b i t s}, D\right)+E_{c p u}+E_{c o n} * R_{c o n}$ Total Energy Spent $=\left(E_{c p u}+E_{c o n} * R_{c o n}\right) *\left(\mathbf{f}_{E R X}\left(T_{b i t s}, D\right)+\right.$ $\left.\mathbf{f}_{E T X}\left(T_{b i t s}, D\right)\right)$

where

$\mathrm{E}_{\mathrm{cpu}}-\mathrm{CPU}, \mathrm{E}_{\mathrm{tx}}-$ Transmission Energy, $\mathrm{E}_{\mathrm{rx}}$ - Receiving Energy , $\mathrm{T}_{\text {bits }}$ - Number of bits transmitted, $\mathrm{E}_{\mathrm{con}}$ - Total energy consumed during connection , $\mathrm{R}_{\text {con }}$ - Total times of re-connection

$\mathbf{f}_{\text {ETX }}\left(T_{\text {bits }}, D\right)$ is a function of energy consumed for transmitting $\mathrm{T}_{\text {bits }}$ of data over distance $\mathrm{D}$. We evaluated the system by calculating the influence of distance over the time taken to deliver the data of sizes 10, 20 and $40 \mathrm{~Kb}$ from the node to the mobile device. The exact value in terms of power consumption is still a work under progress. The results are shown in Figure 9.

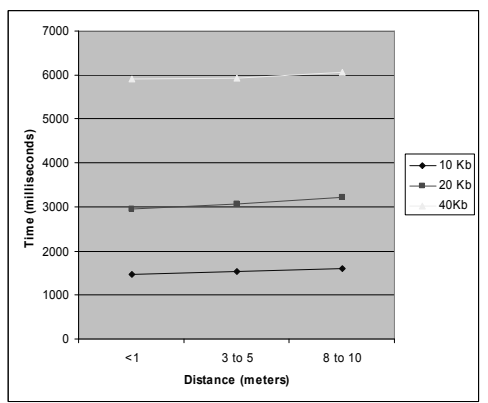

Figure 9: Influence on time over increasing distances \& data sizes

The linear curve represents a gradual increase in time as the distance increases. This is also consistent as the size of the data increases with increased amounts of time for huge amounts of data. Measuring the exact power consumed is a work under progress. We infer from these results that distance does influence the time taken to transfer data and we expect the same with power consumption. Hence reducing the distance of data collection will result in a cost efficient data collection approach.

\section{CONCLUSION AND FUTURE WORK}

The paper has proposed the use of context aware mobile devices as data carriers for sensor data. The mobile nodes have enough spare capacity to form a distributed shared access network that can be used for sensor data collection and delivery. This is made possible with available powerful and context aware mobile devices that can work in pervasive environments like VPAN. The paper has proposed the architectural framework of the components on the mobile node that act as the data carrier. Since the mobile node works within the context aware VPAN space, the node is intelligent enough to arrive at decisions on possible future location and direction of travel as illustrated in the paper. This proposal presents a basic idea behind the usage of context aware mobile devices with spare capacity to build an access network that can be used for various applications.
The preliminary results are encouraging showing how the framework can enable efficient data collection by reducing transmission distance and hence increasing sensor network life. Further work and experimentation is being done to get more accurate results on power consumed and development of a robust accounting model for credit distribution on data delivery.

\section{ACKNOWLEDGMENT}

Thanks to Jens Eliasson of EISLAB, for providing support on the Bluetooth Mulle.

\section{REFERENCES}

[1] Y David Culler, Deborah Estrin, Mani B Srivastava, Overview of sensor networks, IEEE Computer, 2004, vol 37, Pages(s):41-49

[2] Chien-Chung Shen, Chavalit Srisathapornphat, and Chaiporn Jaikaeo. Sensor information networking architecture and applications. IEEE Personal Communications, 2001, Page(s): 5259

[3] R. Shah, S. Roy, S. Jain, W. Brunette, "Data MULEs: Modeling a Three-tier Architecture for Sparse Sensor Networks", IEEE SNPA Workshop, May 2003

[4] K. Aman, A. S. Arun, D. J. David, B. S. Mani, and E. Deborah, "Intelligent fluid infrastructure for embedded networks," in Proceedings of the 2nd international conference on Mobile systems, applications, and services. Boston, MA, USA: ACM Press, 2004.

[5] David Jea, Arun Somasundara, Mani Srivastava, "Multiple Controlled Mobile Elements (Data Mules) for Data Collection in Sensor Networks", International Conference on Distributed Computing in Sensor Systems, 2005

[6] LaMarca, A., Brunette, W., Koizumi, D., Lease, M., Sigurdsson, S. B., Sikorski, K., Fox, D., and Borriello, G. Making Sensor Networks Practical with Robots. In Proceedings of the First international Conference on Pervasive Computing, 2002, Page(s): 152-166.

[7] Shashidhar R. Gandham, M. Dawande, Ravi Prakash and S. Venkatesan, Energy-Efficient Schemes for Wireless Sensor Networks with Multiple Mobile Base Stations. Proceedings of IEEE Globecom, December 2003

[8] Vincze, Z., Vass, D., Vida, R., Vidács, A., and Telcs, A. Adaptive sink mobility in event-driven multi-hop wireless sensor networks. In Proceedings of the First international Conference on integrated internet Ad Hoc and Sensor Networks, 2005

[9] Tong L, Q. Zhao, and S. Adireddy, "Sensor networks with mobile agents," Proc. Of IEEE MILCOM'03, 2003

[10] Ren, B., Ma, J., and Chen, C. The hybrid mobile wireless sensor networks for data gathering. In Proceeding of the international Conference on Communications and Mobile Computing, 2006

[11] A. Chakrabarti, A. Sabharwal, B. Aazhang, Using Predictable Observer Mobility for Power Efficient Design of Sensor Networks, Information Processing in Sensor Networks, 2003

[12] A. A. Somasundara, A. Kansal, D. D. Jea, D. Estrin, and M. B. Srivastava, "Controllably Mobile Infrastructure for Low Energy Embedded Networks," Mobile Computing, IEEE Transactions on, vol. 5, pp. 958-973, 2006.

[13] Jayaraman P. P., Hii P., Zaslavsky A., "Message-On-Demand Service in a Decentralized Unified Messaging System", In WONS 2006 : Third Annual Conference on Wireless On-demand Network Systems and Services, 2006

[14] J. Johansson, M. V“olker, J. Eliasson, `A. “ Ostmark, P. Lindgren, and J. Delsing., "Mulle: A minimal sensor networking device implementation and manufacturing challenges," in IMAPS Nordic 2004, pp. 265-271, 2004.

[15] Östmark A, Jens Eliasson, Per Lindgren, Aart van Halteren, Lianne Meppelink, An Infrastructure for Service Oriented Sensor Networks, JOURNAL OF COMPUTERS, VOL. 1, NO. 5, AUGUST 2006

[16] BlueSoleil, http://www.bluesoleil.com/, January 2006

[17] ZigBee Alliance, http://www.zigbee.org/en/index.asp, January 2006 